\section{P-007 POOR COLLATERALS ON PRE-TREATMENT CT ANGIOGRAPHY PREDICTS POOR OUTCOME AFTER SUCCESSFUL RECANALIZATION IN PATIENTS WITH ANTERIOR CIRCULATION EMERGENT LARGE VESSEL OCCLUSION}

${ }^{1} \mathrm{~N}$ Goyal, ${ }^{1} \mathrm{~S}$ Iftikhar, ${ }^{1} \mathrm{G}$ Tsivgoulis, ${ }^{1} \mathrm{Y}$ Khorchid, ${ }^{2} \mathrm{~A}$ Choudhri, ${ }^{3} \mathrm{D}$ Hoit, ${ }^{1} \mathrm{~A}$ Alexandrov, ${ }^{3} \mathrm{~A}$ Arthur, ${ }^{4} \mathrm{~L}$ Elijovich. ${ }^{1}$ Neurology, University of Tennessee Health Science Center, Memphis, TN; ${ }^{2}$ Radiology, University of Tennessee Health Science Center, Memphis, TN; ${ }^{3}$ Neurosurgery, University of Tennessee Health Science Center, Memphis, TN; ${ }^{4}$ Neurology and Neurosurgery, University of Tennessee Health Science Center, Memphis, TN

\subsection{6/neurintsurg-2016-012589.49}

Background and purpose The best imaging selection technique for endovascular treatment (EVT) remains a topic of debate. Collateral scores (CS) on pre-treatment CT angiography (CTA) have been associated with favorable outcome. We hypothesized that low CS on pre-treatment CTA may predict a poor outcome after EVT in successfully recanalised patients with emergent large vessel occlusions (ELVO).

Methods A retrospective chart review was performed for the University of Tennessee Health Sciences Center Acute Ischemic Stroke Database evaluating AIS patients presenting with CTA confirmed anterior circulation ELVO in a tertiary stroke center during a 3 year period. Only patients with successful recanalization (TICI 2 b or 3 ) after EVT were included in the analysis. A blinded neuroradiologist calculated the CTA CS and final infarct volume (FIV). Poor outcome after EVT was defined as symptomatic intracranial hemorrhage (SICH), cerebral edema requiring hypertonic treatment for $\geq 48$ hours, hemicraniectomy, higher FIV, and poor clinical outcome of modified ranking scale (mRS)-score 3-6 at 3 months.

Results 58 AIS patients with anterior circulation ELVO (mean age $63 \pm 13$ years, $48 \%$ male, median admission NIHSSscore: 17 points, IQR 14-21) had successful recanalization after EVT. Systemic thrombolysis was administered in 38 patients $(65.5 \%)$. A total of 31 patients $(53 \%)$ achieved favorable outcome (FO). There was no significant difference in rates of hemicraniectomy $(\mathrm{p}=1.000)$ and SICH $(\mathrm{p}=0.667)$ after EVT when compared to patients with low and good CS. Patients with low CS tended to have higher rates of cerebral edema requiring hypertonic treatment $(30 \%$ vs $13 \%$, $\mathrm{p}=0.340)$ after EVT. Patients with low CS had greater FIV (111 \pm 71 vs $\left.41 \pm 66 \mathrm{~cm}^{3}, \mathrm{p}=0.007\right)$ and higher rates of poor clinical outcome $(82 \%$ vs $39 \%, \mathrm{p}=0.017)$ in comparison to patients with high CS. A low CS was independently associated with poor clinical outcome $(p=0.048)$ in multiple logistic regression models adjusting for demographics, vascular risk factors, pretreatment SBP, admission NIHSS, intravenous thrombolysis, and onset to revascularization time.

Conclusion Low CS on pre-treatment CTA was correlated with significantly worse outcome despite successful recanalization as evident by higher FIV and higher rates of poor clinical outcome. Poor CS should be considered an important variable in futures trials comparing the medical versus interventional management of patients with ELVO.

Disclosures N. Goyal: None. S. Iftikhar: None. G. Tsivgoulis: None. Y. Khorchid: None. A. Choudhri: None. D. Hoit: None. A. Alexandrov: None. A. Arthur: None. L. Elijovich: None.

\section{P-008 EVALUATING RACIAL/ETHNIC DISPARITY IN ENDOVASCULAR THROMBECTOMY OUTCOME FOR ACUTE STROKE PATIENTS: 4,763 PATIENTS USING PREMIER DATA 2011 TO 2015}

${ }^{1} \mathrm{~S}$ Park, ${ }^{2} \mathrm{M}$ Pilot, ${ }^{3} \mathrm{M}$ Alexander, ${ }^{4} \mathrm{~A}$ Rosengart. ${ }^{1}$ Neuroscience, Albany Medical Center, Albany, NY; ${ }^{2}$ Department of Public Health, Loma Linda University, Loma Linda, CA; ${ }^{3}$ Neurosurgery, Cedars-Sinai Medical Center, Los Angeles, CA; ${ }^{4}$ Neurology, Cedars-Sinai Medical Center, Los Angeles, CA

\subsection{6/neurintsurg-2016-012589.50}

Introduction Prediction of outcome following endovascular thrombectomy in acute stroke is mostly related with the extent of ischemic injury and time treatment window. We were interested in understanding race/ethnicity-related differences in outcome of acute stroke patients who received endovascular thrombectomy with respect to variance in admission demographics and comorbidities during the acute hospitalization phase using the Premier database. Previous studies reported that black patients had significantly lower rates of overall endovascular thrombectomy utilization in the U.S. However, race/ethnicity related outcome in endovascular thrombectomy has not been reported previously.

Methods We utilized the Premier data (2011 to 2015) including 4,763 adults (age $\leq 18$ ) with acute stroke who received endovascular thrombectomy using ICD-9 procedure and chronic/acute comorbidity using ICD-9 diagnostic code for as well as demographic profiles based on administration data. We analyzed outcomes using the patients' ethnicity as independent variables to evaluate racial disparity in endovascular thrombectomy. We identified variables for (1) comorbidities; hypertension, diabetes mellitus, hyperlipidemia, coronary artery disease, congestive heart failure, atrial fibrillation/flutter COPD, tobacco/alcohol dependence and morbid obesity, (2) Socio-economic status; age, sex and races/ethnicities were categorized as binary variables as white versus non-white and black versus non-black and hispanic versus non-hispanic. Chi-square analysis used for binary independent factor race variables. Race variables categorized as (1) white, (2) black, and (3) others. The Bonferroni correction was applied to compare means among groups. We compared in-hospital outcome including mortality, post-operative stroke and functional outcome using discharge disposition; home versus transferred to other hospital or not adjusting acute and chronic comorbidity using multiple logistic regression (SAS 9.4).

Results The mean age for endovascular thrombectomy patients with acute stroke was 66.8 years (SD \pm 15.4 years) with $58 \%>65$ years old; $51.2 \%$ females; $70.7 \%$ whites, $12.1 \%$ blacks, $0.2 \%$ hispanics and $17 \%$ others. Black and hispanic races received relatively low rate of endovascular thrombectomy utilization compared to white $(\mathrm{p}=0.001)$. As comorbidities, 76.1\% had HTN, 33.2\% DM, 50.3\% HLD, 28\% CAD, 20.7\% CHF, A fibrillation or flutter 44.9\%, 2\% COPD, $12.2 \%$ chronic renal failure, $10.5 \%$ acute renal failure, $18 \%$ tobacco dependence, $1.5 \%$ alcohol dependence, $4.2 \%$ morbid obesity, 2.3\% Pneumonia, 5.0\% Sepsis and 5.2\% MI. As an outcome makers, in-patient mortality $16.7 \%$ (white $16.8 \%$, black $14.9 \%$, hispanic $0 \%$ and others $17.6 \%$ ), discharge to home $28.7 \%$ (white 28\%, black 31.4\%, hispanic 22.2\% and others $30 \%$ ) versus disposition to any facility $71.3 \%$ (white $72 \%$, black 69.6\%, hispanic $77.8 \%$ and others $70 \%$ ), and post thrombectomy stroke was $6.3 \%$ (white $6.5 \%$, black $5.4 \%$, hispanic $0 \%$ and others $6.3 \%$ ). Since number of hispanics is very small, we divided the cohort into three racial groups: 
white, black, and others. Multiple variables for chronic/acute comorbidities identified associated with outcomes. After adjustment using logistic regression, race was not associated with three in-hospital outcome variables: mortality $(\mathrm{p}=0.183)$, post-operative stroke $(\mathrm{p}=0.610)$ and discharge disposition $(\mathrm{p}=0.231)$.

Conclusions There were no differences in hospital outcome among races/ethnicities in endovascular thrombectomy outcomes for acute stroke patients in the Premier data. Racial/ ethnic disparities play role for patients' selection not for patients' outcome in endovascular thrombectomy.

Disclosures S. Park: None. M. Pilot: None. M. Alexander: 1; C; Consultant for Stryker Neurovascular, Medtronic, and Penumbra, Inc. A. Rosengart: None.

\section{P-009 ASPECTS DECAY DURING INTER-FACILITY TRANSFER IN PATIENTS WITH LARGE VESSEL OCCLUSION STROKES AND ITS IMPACT ON ELIGIBILITY FOR ENDOVASCULAR PROCEDURES}

${ }^{1} \mathrm{M}$ Mokin, ${ }^{2} \mathrm{R}$ Gupta, ${ }^{1} \mathrm{~W}$ Guerrero, ${ }^{1} \mathrm{D}$ Rose, ${ }^{1} \mathrm{~W}$ Burgin, ${ }^{1} \mathrm{~S}$ Sivakanthan. ${ }^{1}$ University of South Florida, Tampa, FL; ${ }^{2}$ Wellstar Neurosurgery, Marietta, GA

\subsection{6/neurintsurg-2016-012589.51}

Background Favorable imaging profile according to the Alberta Stroke Program Early CT Score (ASPECTS) on noncontrast head CT is a key criterion for the selection of patients with ischemic stroke from large vessel occlusion (LVO) for intraarterial (IA) revascularization therapies.

Objective The goal of our study was to analyze factors associated with changes in ASPECTS during inter-hospital transfer and to determine their impact on eligibility for endovascular procedures.

Methods We analyzed factors associated with changes in ASPECTS during inter-hospital transfer and their potential impact on eligibility for IA stroke therapies in patients with anterior circulation ischemic strokes. Clinical and demographic characteristics between patients with favorable and unfavorable imaging on repeat CT were compared. Favorable ASPECTS profile was defined as ASPECTS $\geq 6$, and unfavorable ASPECTS $<6$, based on the imaging criteria proposed by the AHA in the 2015 updated acute stroke guidelines.

Results Of the 50 transferred patients with anterior circulation LVO, 42 had favorable ASPECTS $\geq 6$ on CT imaging performed at outside hospital. 19 (45\%) of those 42 patients presented to an outside facility within 6 hours of stroke onset (mean time from symptom onset to head CT, $295 \pm 61 \mathrm{~min}$ ), whereas in 23 patients CT showed favorable ASPECTS with stroke onset beyond the 6 hour window (mean time from symptom onset to outside CT, $603 \pm 224 \mathrm{~min}$ ). Stroke evolution towards unfavorable ASPECTS occurred in 13 (31\%) out of 42 patients who initially had favorable imaging profile at outside hospitals. Higher NIHSS score was the only significant predictor of ASPECTS decay, whereas other clinical characteristics such as the use of intravenous thrombolysis and site of LVO (ICA versus MCA M1/M2) were similar between both groups.

Conclusions Our study showed that during inter-hospital transfer, one out of three patients with stroke from anterior circulation LVO becomes ineligible for IA thrombectomy based on CT ASPECTS imaging criteria alone. Except for NIHSS severity, no other baseline clinical factors could identify which patients were at risk of ASPECTS deterioration. Our study indicates the critical importance of rapid transfer of all stroke patients with suspected LVO to endovascular-capable hospitals. Disclosures M. Mokin: None. R. Gupta: 1; C; Zoll, WellStar foundation. 2; C; Stryker Neurovascular, Covidien, Penumbra, Rapid medical. 6; C; Penumbra, Inc. W. Guerrero: None. D. Rose: 3; C; Boehringer Ingelheim Pharmaceuticals, ChiesiUSA. W. Burgin: None. S. Sivakanthan: None.

\section{P-010 ASSOCIATION OF CLOT BURDEN SCORE WITH RADIOGRAPHIC AND CLINICAL OUTCOMES FOLLOWING SOLITAIRE STENT RETRIEVER THROMBECTOMY: ANALYSIS OF THE SWIFT PRIME TRIAL}

${ }^{1} \mathrm{M}$ Mokin, ${ }^{2} \mathrm{E}$ Levy, ${ }^{2} \mathrm{~A}$ Siddqui, ${ }^{3} \mathrm{M}$ Goyal, ${ }^{4} \mathrm{R}$ Nogueira, ${ }^{5} \mathrm{P}$ Yavagal, ${ }^{6} \mathrm{~V}$ Pereira, ${ }^{7} \mathrm{~J}$ Saver. ${ }^{1}$ University of South Florida, Tampa, FL; ${ }^{2}$ University at Buffalo, Buffalo, NY; ${ }^{3}$ University of Calgary, Calgary, AB, Canada; ${ }^{4}$ Emory University, Atlanta, GA; ${ }^{5}$ University of Miami, Miami, FL; ${ }^{6}$ University of Toronto, Toronto, ON, Canada; ${ }^{7}$ University of California Los Angeles, LoS Angeles, CA

\subsection{6/neurintsurg-2016-012589.52}

Background Clot burden score (CBS) was developed as a tool to evaluate the extent of intracranial thrombus burden in patients with anterior circulation acute ischemic stroke. Its value in predicting radiographic and clinical outcomes in patients treated with endovascular stroke therapy remains unknown.

Objective To evaluate the relationship between CBS and outcomes after stent retriever thrombectomy in the interventional arm of the SWIFT PRIME trial.

Methods CBS was calculated for the endovascular arm (intravenous tPA plus Solitaire stent retriever) of SWIFT PRIME using baseline CTA. The cohort of 69 patients was divided into 3 groups according to their CBS values: CBS 0-5 $(\mathrm{n}=14)$, CBS 6-7 $(\mathrm{n}=23)$ and CBS 8-9 $(\mathrm{n}=32)$. Association between CBS and outcomes following treatment with the Solitaire device was studied.

Results The mean age of the 69 patients who formed the study cohort was $63.2 \pm 13.1$, mean NIHSS score was $16.8 \pm 4.5$, and $55 \%$ were males. There was no difference in clinical characteristics among the 3 groups, except for the baseline ASPECTS ( $\mathrm{P}=0.049$ ). The site of proximal occlusion varied significantly among the 3 groups $(\mathrm{P}<0.001)$. Rates of successful recanalization (TICI $2 \mathrm{~b} / 3$ ), complete recanalization (TICI 3 only), and of good clinical outcome at 3 months were similar among the 3 groups. $(\mathrm{P}=0.24$, $\mathrm{P}=0.35$, and $\mathrm{P}=0.52$, respectively).

Conclusions The combination of IV thrombolysis and stent retriever thrombectomy with the Solitaire device is highly effective in achieving successful recanalization and a good clinical outcome throughout the entire range of CBS values.

Disclosures M. Mokin: None. E. Levy: 4; C; Intratech Medical Ltd., Blockade Medical LLC, Medina Medical. 6; C; Covidien (Medtronic), Abbott, Intratech Medical and Blockade Medical. A. Siddqui: 2; C; Codman \& Shurtleff, Inc., Concentric Medical, ev3/Covidien Vascular Therapies, GuidePoint Global Consulting, Penumbra, Stryker, Pulsar Vascular, MicroVention, Lazarus Effect, Blockade Medical. 3; C; Codman \& Shurtleff, Inc.. 4; C; Hotspur, Intratech Medical, StimSox, Valor Medical, Blockade Medical, and Lazarus Effect. M. Goyal: 1; C; Covidien. 6; C; Covidien. R. Nogueira: 2; C; Stryker. 6; C; Covidien. D. Yavagal: 6; C; Covidien. V. Pereira: 2; C; Medtronic Neurovascular, Stryker. J. Saver: 1; C; 